\title{
Mobile Interaction Using Paperweight Metaphor
}

\author{
Itiro Siio \\ Faculty of Science \\ Ochanomizu University \\ 2-1-1 Otsuka \\ Bunkyo-ku, Tokyo 112-8610 Japan \\ $+81-3-5978-5398$ \\ siio@acm.org
}

\author{
Hitomi Tsujita \\ Graduate School of Humanities and \\ Sciences \\ Ochanomizu University \\ 2-1-1 Otsuka \\ Bunkyo-ku, Tokyo 112-8610 Japan \\ gü220529@edu.is.ocha.ac.jp
}

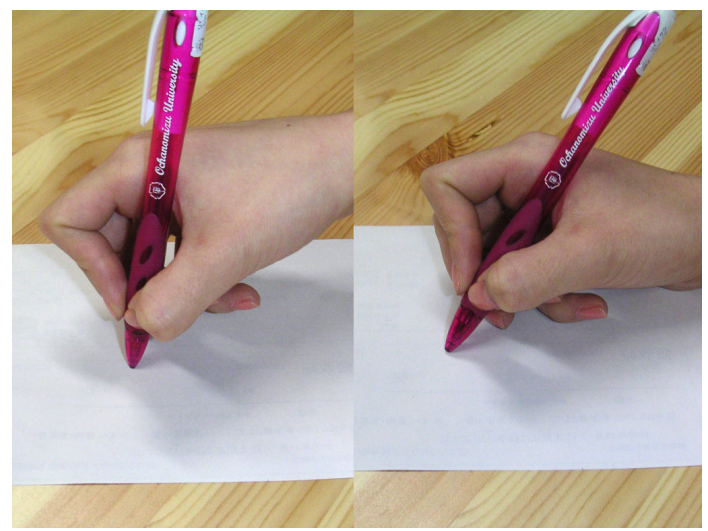

\begin{abstract}
Conventional scrolling methods for small sized display in PDAs or mobile phones are difficult to use when frequent switching of scrolling and editing operations are required, for example, browsing and operating large sized WWW pages.

In this paper, we propose a new user-interface method to provide seamless switching between scrolling and other operations such as editing, based on "Paperweight Metaphor". A sheet of paper that has been placed on a slippery table is difficult to draw on. Therefore, in order to write or draw something on the sheet of paper, a person must secure the paper with his/her palm to avoid the paper from moving. This will be a good metaphor to design switching operation of scroll and editing modes.
\end{abstract}

We have made prototype systems by placing a touch sensor under each PDA display where user's palm will be hit. Three application programs - map browser, WWW browser, and photograph browser - that switch between scrolling and other operation modes depending on sensor output have been developed. We have carried out user tests on this mode switching method and have received favorable feedback on the same.

ACM Classification: H5.2 [Information interfaces and presentation]: User Interfaces. - Input devices and strategies.

General terms: Design, Human Factors.

Keywords: Paperweight metaphor, input devices, mobile devices, pen interface, small display, touch sensor, scroll.

\section{INTRODUCTION}

In miniature handheld computing devices such as PDAs, mobile phones, displays are small, and there are many limitations and difficulties to indicate over sized contents at once. In general, only a part of the contents is shown at once, and other part of the contents can only be shown by a scrolling operation. There are many user-interface techniques to display desired part of contents by scrolling and/or zooming.

Permission to make digital or hard copies of all or part of this work for personal or classroom use is granted without fee provided that copies are not made or distributed for profit or commercial advantage and that copies bear this notice and the full citation on the first page. To copy otherwise, to republish, to post on servers or to redistribute to lists, requires prior specific permission and/or a fee.

UIST'06, October 15-18, 2006, Montreux, Switzerland..

Copyright 2006 ACM 159593313 1/06/0010 ... \$5.00.
Figure 1: Explanation of "Paperweight Metaphor." Since the paper on the desk moves when writing tool is moved (left), we must hold it down it by our hand to write letters on it (right). Making use of the familiar action as a metaphor, we could design intuitive switching operation for the scroll and edit modes.

One of the most common techniques is to place scroll bars in the edge of the display. Another popular method is a scroll mode that enables a user to scroll displayed contents by dragging it with a pen device. Conventional scrolling methods require the precise selection of scroll bars or buttons by the user. They are difficult to use, especially when a user wants to frequently switch between scrolling and content modification such as editing. He/she has to access scroll bars or buttons to switch to scroll mode while browsing WWW contents or writing / drawing documents that were designed originally for large sized displays.

A new user-interface method to provide seamless switching of scrolling, zooming, and editing modes, has been desired for handheld computing devices.

\section{PAPERWEIGHT METAPHOR}

We propose a new user-interface method to provide seamless and intuitive switching of scrolling mode and editing mode, based on "Paperweight Metaphor".

When we place a sheet of paper on a slippery tabletop as shown in fig. 1, and try to write letters on the paper with one 


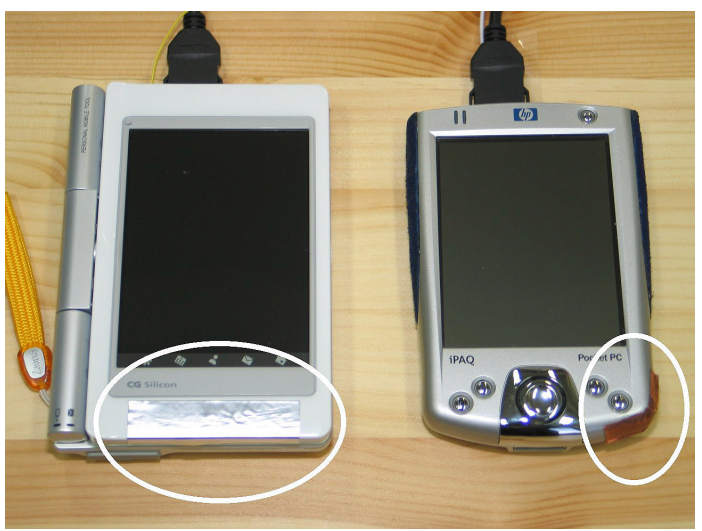

Figure 2: There are two kinds of prototype systems. Left prototype has a touch sensor electrode (marked by white circle) in the bottom, while right has it at the right-bottom corner. Right-handed users preferred the right one.

hand, we have to fix it by our palm (fig. 1 right), because it will be slipped following to the movement of the pen (fig. 1 left).

This familiar writing action of using our hand as paperweight to fix a sheet of paper is a good metaphor to design switching operation of scroll and editing modes. We can design new interaction methods using the Paperweight Metaphor. For example, the action of sliding a sheet of paper can be used to scroll contents in a computer display, and the action of writing letters by fixing it can be used to edit the contents.

The Paperweight Metaphor can also be applied to other operations, i.e. rotation and zooming operations. When a user moves the pen from left to right, while he/she is softly pressing the sheet of paper with his/her palm, it may be rotated clockwise around the place where his/her palm is attaching. This action can be mapped to rotation operations. Assume that the sheet of paper is made of elastic material such as a thin rubber-sheet, pressing against the sheet by one's palm and moving the pen upward may stretch the sheet. Conversely, moving the pen downward may cause the sheet to shrink. This action can be mapped to zooming in and zooming out operations.

\section{APPLICATIONS}

To realize the interaction using the Paperweight Metaphor, we have made two kinds of prototype systems by placing a touch sensor ${ }^{1}$ electrode under each PDA ${ }^{2}$ display where user's palm will be hit (fig. 2). The silver electrode under the palm is the sensing area as shown in fig. 3. The output of the touch sensor is wired to the PDA. By detecting whether a user's palm is touching the sensor or not, the mode of pen operation changes. In order to assess our interaction method, we have developed three application programs ${ }^{3}$, that is, map browser (fig. 3), WWW browser and photograph browser (fig. 4).

\footnotetext{
${ }^{1}$ Quantum Research Group: QTouch.

${ }^{2}$ SHARP: Zaurus SL-C720 (Linux OS) and Hewlett Packard: iPAQ h2210 (Windows Mobile 2003).

${ }^{3}$ Written in Java (for Zaurus) and C\# (for iPAQ).
}
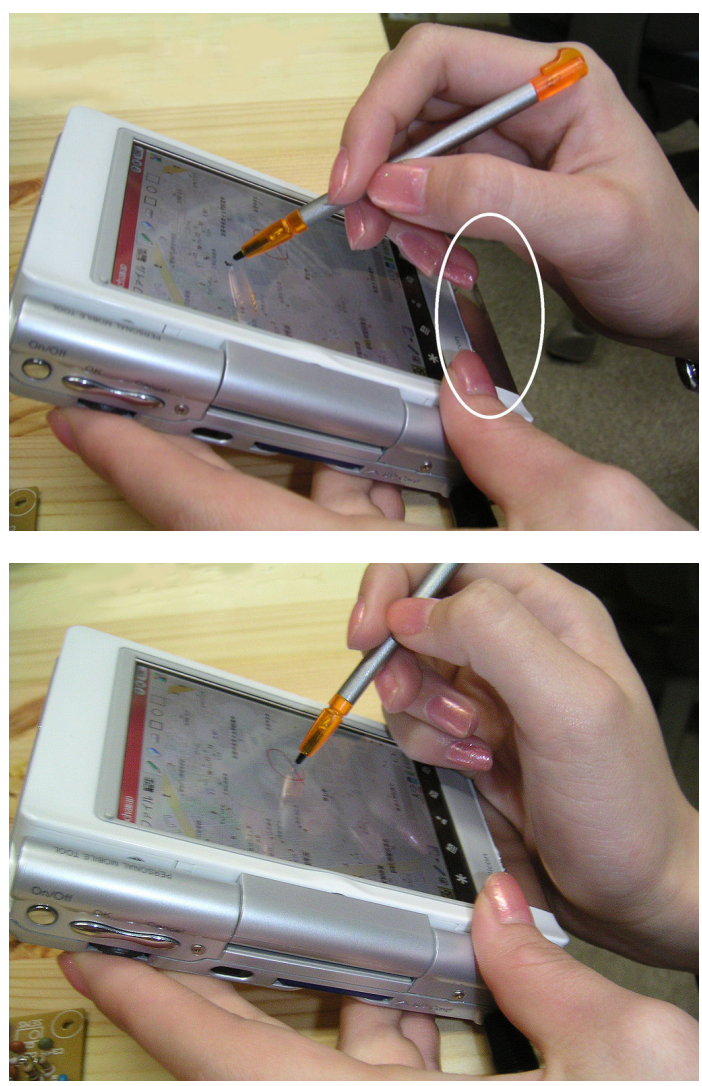

Figure 3: (top) A user scrolls the map while not touching the sensor (at the oval mark) and drugging. (bottom ) A user draws handwritten note while touching the sensor.

\section{Map Browser}

Fig. 3 shows a map browser and annotator application that we have developed for demonstration purposes. When a user does not touch his/her palm from the touch sensor, and drags the map by using the pen device, the map scrolls according to the movement of the pen. When a user touches the sensor by his/her palm, the map does not scroll and a user can draw handwritten annotations while dragging the pen on the map.

\section{WWW Browser}

We have developed a WWW browser application based on the Paperweight Metaphor. As same as in the previous application, when a user is not touching the sensor, he/she can scroll WWW pages in any directions by dragging the pen device on the display. On the other hand, when a user touches the sensor, he/she can manipulate the WWW pages in a normal web-browsing manner. He/she can click on linked texts and images, can enter characters in text entry fields by using a software keyboard or character recognition boxes, and can press buttons on the WWW page.

\section{Photograph Browser}

Fig. 4 shows a photograph browser application. In this application, when a user's hand is not touching the sensor, the user can scroll the photograph using the pen device, on the other hand, when a user's hand is touching the sensor, the user can zoom and rotate the photograph using the pen de- 


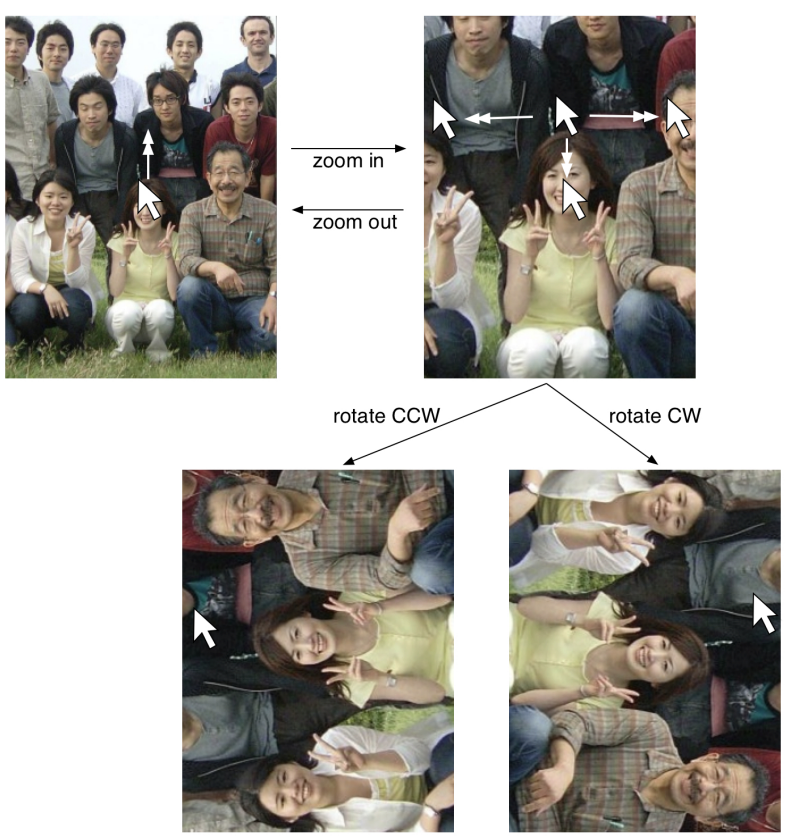

Figure 4: Zooming in/out and rotating a photograph by pen dragging while touching the sensor.

vice. This is another phenomenon that may occur in the $\mathrm{Pa}$ perweight Metaphor as mentioned in the previous section. That is, dragging the pen upward, allows the photograph to stretch and zoom-in operation is invoked, and dragging the pen downward invokes zoom-out operation. Dragging the pen left to right invokes clockwise rotation, and right to left invokes counter clockwise rotation. By using these operations, a user can browse the photograph easily by scrolling, zooming in/out, and rotating seamlessly.

\section{Other Potential Applications}

Apart from the abovementioned applications, we can develop a word processor or a graphic editor that enables switching between the scrolling, text-editing, or drawing modes by using the touch sensor operations. Many business PDA applications such as a medical records viewer and annotator and an on-site inventory system could be possible. In wearable computing applications, hands-free operation is often required. Many wrist-mounted computers, displays, and input devices are developed such that they can be operated by using only one hand. ${ }^{4}$ The one-hand operation capabilities by the Paperweight Metaphor are useful for these types of wearable input devices.

\section{USER STUDY \\ User Feedback}

We have carried out user study to find the optimal position of the touch sensor electrode. For this purpose, we have used one of our prototypes that has a wide electrode under the display as shown in the left in fig. 2. The subjects are 7 undergraduate students, who are around 22 years old and not regular PDA users. Two of them are left-handed. The map browser application described in the previous section

\footnotetext{
${ }^{4}$ Such as Symbol Technologies: WSS1000 and http://www.orang otang.com/
}

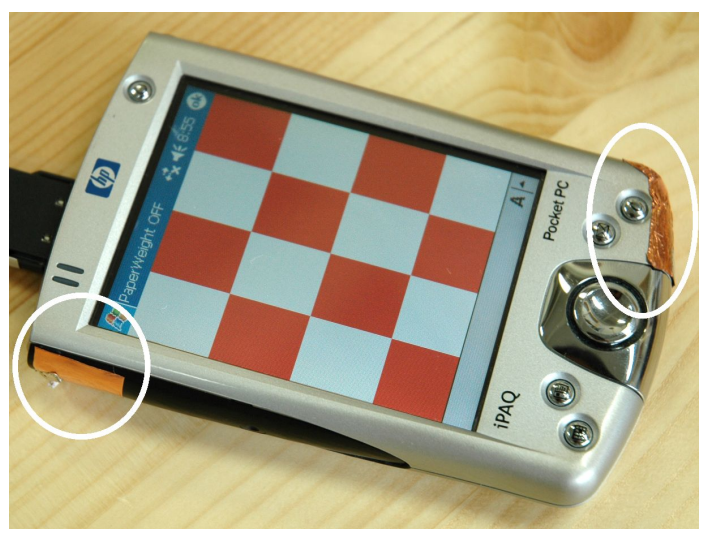

Figure 5: A prototype for the comparison experiment with two sensor electrodes: one for the palm of the dominant hand (at the right oval mark) and the other for the thumb of the non-dominant hand (left).

was running on the PDA and showed maps around their university campus. They were asked to draw a route on the map, from the nearest subway station to the university entrance gate. The task requires frequent switching of scrolling and drawing operations using the touch sensor.

We have studied their action and found out that most of the subjects have been using their dominant-hand-side part of the sensor electrode. All of our right-handed subjects prefer to use the prototype that has the sensor electrode at the bottom right corner which is shown in the right in fig. 2 . We can conclude that bottom corner is a good place to install the touch sensor, however, further adjustment on sensor size and position will be required depending on the size and shape of the PDA and the applications.

Following above user study, we have demonstrated the prototype with a right-corner sensor (right one in fig. 2) to more than 50 participants at conferences. We have received many favorable comments that the mode switching operation based on the Paperweight Metaphor is intuitive and easy to use and learn. Some of them have pointed out that it is difficult to edit objects at the bottom-right corner of the display, which is near the touch sensor. They requested an additional touch sensor where they can place their non-dominant hand. Prior study[5] also suggests that placing a switch button for the non-dominant hand to press on will improve performance.

\section{Comparison}

We have compared the performance of our method with two other methods - one involves the use of conventional scroll bars and the other involves switching with the non-dominant hand that is described in the previous section. To enable this comparison, we have developed a prototype with two touch sensor electrodes - one for the palm of the dominant hand and the other for the thumb of the non-dominant hand (fig. 5). A mechanical switch is used to connect one of the electrodes to the touch sensor module.

Fig. 5 also shows our experimental program that displays $100(10 \times 10)$ red and white rectangles of $60 \times 70$ pixels in a checkerboard pattern. The color of each rectangle chenges to 
white or red when tapped with a stylus. As the display area of the PDA ${ }^{5}(240 \times 268$ pixels $)$ is smaller than the total size of the rectangles $(600 \times 700$ pixels $)$, we have to scroll in order to view all of them. We have developed programs for three types of scroll methods that involve the use of (1) the palm sensor, (2) the thumb sensor, and (3) vertical and horizontal scroll bars of 13 pixels width. Eight subjects aged from 22 to 50 ( 3 men and 5 women), who were not regular PDA users, participated in this experiment. They were asked to change the color of all the 50 white rectangles to red. Each of the three scrolling methods were performed twice, and the order in which they were performed were reversed the second time.

Table 1: Average completion time (sec.). (Errors)

\begin{tabular}{c|c|c|c}
\hline & Palm sensor & Thumb sensor & Scroll bars \\
\hline 1st trial & $40.0(1.0)$ & $47.1(1.1)$ & $59.4(4.3)$ \\
2nd trial & $37.4(1.1)$ & $36.1(0.9)$ & $48.6(3.0)$ \\
\hline
\end{tabular}

Table 1 shows the average completion time and the number of taps on the red rectangles in parentheses. Since tapping a red rectangle is not necessary for the task, we interpreted that it was caused by an unsuccessful scrolling action. Our method, using the palm sensor, is superior to the scroll bars both in terms of speed and error counts. On the other hand, the difference between the palm and thumb sensor is minimal. Although some of the subjects also complained of difficulty in selecting rectangles near the palm sensor, all of them favored it. In comparison to the palm sensor, many of the subjects claimed that the mapping to the scrolling and tapping modes in the thumb sensor method is arbitrary; they also complained that it was sometimes difficult to realize the current mode without practice. The result that the palm sensor is faster than the thumb sensor in the 1st trial may reflect the mode confusion caused by the unfamiliar mapping in the thumb sensor method.

\section{RELATED WORKS}

Making use of the user's fingertip to touch the pad device of laptop computers, the ThumSense system has provided additional mouse-button-functions to some of the keys in the QWERTY keyboard [8]. In our research, we have also focused on designing seamless mode switching with the contact of the human body.

Various approaches for incorporating our natural body movements into portable handheld devices have been proposed. Interaction techniques are proposed by arranging fingertip contact position sensors around the display of the portable computer [3]. A user can turn pages by stroking one of the sensors. Rekimoto [7] and Fitzmaurice [1] used tilt sensor for navigating maps and menus. Harrison [2] used pressure and tilt sensors for scrolling documents and lists. RodDirect [6] used interaction technique for handheld computers by rotating and sliding a pen device in a pen holder of the computer body. We have focused on smooth switching of scroll mode based on the Paperweight Metaphor and realized it by using a simple touch sensor.

\footnotetext{
${ }^{5}$ Hewlett Packard: iPAQ h2210.
}

The rotating and zooming techniques used in our photograph browser application are similar to those for bimanual interaction[4]. Our method uses a palm and fingers holding a pen, instead of using two hands.

\section{SUMMARY AND FUTURE WORKS}

In this paper, we have proposed new interaction techniques based on the Paperweight Metaphor. We have developed three prototype applications to show that this metaphor provides smooth operation for handling large contents. Using our prototype systems, we have carried out user tests and received useful feedbacks. We are planning to implement further applications based on the Paperweight Metaphor for mobile phones, palm top, tablet, notebook and wearable computers.

\section{REFERENCES}

1. George W. Fitzmaurice. Situated information spaces and spatially aware palmtop computers. Commun. ACM, 36(7):39-49, 1993.

2. Beverly L. Harrison, Kenneth P. Fishkin, Anuj Gujar, Carlos Mochon, and Roy Want. Squeeze me, hold me, tilt me! an exploration of manipulative user interfaces. In CHI '98: Proceedings of the SIGCHI conference on Human factors in computing systems, pages 1724, New York, NY, USA, 1998. ACM Press/AddisonWesley Publishing Co.

3. Ken Hinckley, Jeff Pierce, Mike Sinclair, and Eric Horvitz. Sensing techniques for mobile interaction. In UIST '00: Proceedings of the 13th annual ACM symposium on User interface software and technology, pages 91-100, New York, NY, USA, 2000. ACM Press.

4. Celine Latulipe, Craig S. Kaplan, and Charles L. A. Clarke. Bimanual and unimanual image alignment: an evaluation of mouse-based techniques. In UIST '05: Proceedings of the 18th annual ACM symposium on User interface software and technology, pages 123-131, New York, NY, USA, 2005. ACM Press.

5. Yang Li, Ken Hinckley, Zhiwei Guan, and James A. Landay. Experimental analysis of mode switching techniques in pen-based user interfaces. In $\mathrm{CHI}$ '05: Proceedings of the SIGCHI conference on Human factors in computing systems, pages 461-470, New York, NY, USA, 2005. ACM Press.

6. Motoki Miura and Susumu Kunifuji. Using stylus as a peripheral input device. In ACM UIST2005 Companion (Demo), pages 45-46, 2005.

7. Jun Rekimoto. Tilting operations for small screen interfaces. In UIST '96: Proceedings of the 9th annual ACM symposium on User interface software and technology, pages 167-168, New York, NY, USA, 1996. ACM Press.

8. Jun Rekimoto. ThumbSense: automatic input mode sensing for touchpad-based interactions. In $\mathrm{CHI}$ '03: CHI '03 extended abstracts on Human factors in computing systems, pages 852-853, New York, NY, USA, 2003. ACM Press. 